\title{
Anomalous properties of hippocampal lesion-induced retrograde amnesia
}

\author{
CANTEY LAND, MICHAEL BUNSEY, and DAVID C. RICCIO \\ Kent State University, Kent, Ohio
}

\begin{abstract}
Retrograde amnesia can result from transient or permanent insults to the central nervous system and is typically manifest as a temporally graded memory loss. This temporal gradient of retrograde amnesia has been considered evidence for memory consolidation, since newly acquired information is vulnerable to amnestic treatments, whereas older information is not. Although investigations of transient insult-induced retrograde amnesia have provided evidence against a consolidation interpretation, hippocampal lesion-induced retrograde amnesia is still considered to represent a consolidation (or storage) failure. In order to investigate the consolidation interpretation of hippocampal lesion-induced retrograde amnesia, two experiments were undertaken. In the first, rats were reminded of "old" memories prior to hippocampal lesions, a procedure that produced retrograde amnesia. This result is difficult to reconcile with current interpretations of retrograde amnesia, since consolidated memories are presumably not vulnerable to amnesia. The second experiment explored the permanence of hippocampal lesioninduced retrograde amnesia by presenting amnestic rats with a portion of the training treatment (reactivation) prior to testing. The reactivation treatment successfully reversed retrograde amnesia. Taken together, the results from these experiments indicate that hippocampal retrograde amnesia may not in all cases arise from storage failure and illuminate new circumstances under which damage to the hippocampus may affect memory.
\end{abstract}

Insults to the central nervous system (CNS) can result in amnesia, or the inability to recall learned information. When the information was learned prior to the insult, the resulting deficit is referred to as retrograde amnesia. This manifestation of amnesia results from two broad classes of experimental treatments: transient disruptions of CNS functioning or permanent damage to brain tissue. Transient insults include such treatments as electroconvulsive shock (ECS; Duncan, 1949), thermoregulatory disruption, concussive head injury, and electrical brain stimulation (see, e.g., Bures \& Buresova, 1963; Kesner \& Doty, 1968; Patterson, Lawler, \& Rochester, 1978; Riccio, Hodges, \& Randall, 1968). These manipulations exert a short-acting or transient impact on CNS function; after the treatment, apparently normal brain functioning is evident, although the amnesia persists. Retrograde amnesia also results from permanent CNS tissue damage, especially that targeting the hippocampal system (Squire, 1992). Unlike transient amnestic treatments, lesion treatments are not reversible

This manuscript is dedicated to the memory of Mike Bunsey. These experiments represent partial fulfillment of the requirements for the doctoral degree at Kent State University for the first author. This research was supported by NIMH MERIT Grant 37535 to D.C.R. and NIMH FIRST Award 1R29NS36962-01 to M.B. We thank John Land, Jr. for Y-maze construction and Stefan Ursu, M.D., for extensive support and assistance. C.L. is now at the University of Kentucky, Department of Pharmacology and Tobacco and Health Research Institute. Correspondence concerning this article should be addressed to D. C. Riccio, Department of Psychology, Kent State University, Kent, OH 44242-0001 (e-mail: driccio@kent.edu). conditions. They permanently modify CNS functioning, and the brain is unlikely to return to a prelesion state.

Both transient and permanent treatments produce retrograde amnesia that is temporally graded (Ribot, 1882), in that amnesia is evident for information learned shortly before the treatment, but not for information learned long before treatment. This gradient has been explained in terms of memory consolidation, a hypothetical process by which new information is transferred from temporary to permanent storage, perhaps by a process akin to the reverberatory circuits once proposed by Hebb (1949). When consolidation of information is complete, the memory is no longer susceptible to amnestic treatments. According to this model, amnesia results from the disruption of the consolidation process. Amnestic treatments interrupt the permanent storage of new information but leave previously stored memories unaffected (McGaugh, 1966; Squire, 1992; Squire \& Alvarez, 1995).

Despite the proficiency with which consolidation theory accounts for the temporal gradient of retrograde amnesia resulting from both transient and permanent $\mathrm{CNS}$ insults, this model has been challenged in studies of transient insult-induced amnesia. Misanin, Miller, and Lewis (1968) noted that the age of a memory is not the only factor that varies between new and old memories: The activity state of the memory presumably varies as well. That is, new memories are more likely to be actively processed than old ones, and this factor could result in differential susceptibility to amnesia. Operationally, reexposure to cues associated with training is considered to reactivate an old but otherwise intact memory. Although there is no known 
independent assessment of the activity status of memory following a reactivation treatment, a number of outcomes are consistent with the concept that old information, which would not be affected by an amnestic agent, becomes susceptible again following such cuing manipulations. Thus, Misanin et al. designed an experiment in which the age of a memory was held constant while its activity state was varied. Briefly, rats were trained to suppress drinking to a signal that predicted footshock. Twentyfour hours later, some subjects received an ECS treatment. After such a delay, ECS does not usually produce amnesia, since the memory is presumably consolidated. In their experiment, however, one group of rats was reminded of the task by an exposure to the conditioned stimulus, or predictor of shock, immediately prior to the amnestic treatment. Rats did not exhibit amnesia if their memories were inactive at the time of ECS. If, however, the reminder treatment immediately preceded ECS, amnesia resulted. The authors concluded that the activity state of a memory, rather than its age, determines vulnerability to retrograde amnesia, and this result has been replicated with other treatments and tasks (e.g., Mactutus \& Riccio, 1978). Such results do not appear consistent with the notion that amnesia results from an interruption of a postacquisition consolidation process of limited duration.

Although a consolidation-disruption view of amnesia is no longer considered a tenable explanation for retrograde amnesia induced by ECS, hypothermia, or other transient insults, this explanation remains the commonly accepted one for retrograde amnesia that results from hippocampal lesions (McClelland, McNaughton, \& O'Reilly, 1995; Moscovitch, 1995; Squire, 1992; Squire \& Alvarez, 1995; Squire \& Zola-Morgan, 1990). Largely on the basis of studies demonstrating temporally graded retrograde amnesia, a number of prominent theories of hippocampal function interpret it as a temporary storage site that consolidates information into extra-hippocampal stores over time following learning. New memories, still dependent on the hippocampus and not yet fully consolidated, are vulnerable to its damage, whereas older, consolidated memories are not (McClelland et al., 1995; Squire, 1992; Squire \& Kandel, 1999; Squire \& ZolaMorgan, 1990; Zola-Morgan \& Squire, 1992). However, in many investigations of amnesia, the age and activity state of memory are confounded. Whether this important theoretical consideration is relevant to amnesia resulting from hippocampal damage is not known. The following experiments represent an attempt to evaluate the effect of activity state of memory on induction and expression of hippocampal lesion-induced amnesia.

\section{EXPERIMENT 1}

Investigations with humans (Scoville \& Milner, 1957), nonhuman primates (Squire \& Zola-Morgan, 1990; ZolaMorgan \& Squire, 1992), rabbits (Kim, Clark, \& Thompson, 1995), and rats (Anagnostaras, Maren, \& Fanselow,
1999; Kim \& Fanselow, 1992; Maren, Aharonov, \& Fanselow, 1997; Winocur, 1990) have demonstrated temporally graded retrograde amnesia following hippocampal damage. In those experiments in which time of exposure to information was controlled (Anagnostaras et al., 1999; Kim et al., 1995; Kim \& Fanselow, 1992; Maren et al., 1997; Squire \& Zola-Morgan, 1990; Winocur, 1990; Zola-Morgan \& Squire, 1992), results indicated that memories formed long (several weeks or more) before surgery were unaffected by lesions, whereas memories formed shortly (about 3 weeks or fewer) before surgery were disrupted.

In each of these cases, however, it is possible that the activity state of the memory varied along with its age, with recently formed memories more apt to be in an active state than old memories. To determine the effect of activity state on vulnerability of memory to hippocampal amnesia, a paradigm conceptually similar to that of Misanin et al. (1968) was used. Rats were trained in a signaled avoidance Y-maze task (Gisquet-Verrier \& Schenk, 1994; Silveria \& Kimble, 1968), in which they were required to run to the lighted arm of a three-arm apparatus, initially to escape and later to avoid mild footshock. This task was chosen because rats with hippocampal damage are not impaired at learning it (Gisquet-Verrier \& Schenk, 1994; Silveria \& Kimble, 1968), making it possible to separate the retrograde and the anterograde effects of the lesion. Because memory is assessed in terms of relearning or savings, it is critical that the lesion itself does not alter the baseline acquisition rate. Furthermore, the task is rapidly acquired, requiring approximately 15 training trials to learn (rats are trained to a criterion of 3 consecutive correct trials), all of which are completed within one episode of about $30 \mathrm{~min}$. Episodic tasks are most vulnerable to hippocampal damage and are less likely to recruit procedural memory systems (Cohen, 1984). Furthermore, the hippocampal memory system is considered crucial for episodic memory (see Cohen \& Eichenbaum, 1993). It was assumed that retrograde amnesia for this signaled avoidance task would occur because of its episodic nature, rather than because of the task demands themselves. Although retrograde and anterograde deficits typically mirror one another, the ability to remember an episode differs from the ability to learn it. We selected a task that hippocampal rats are able to perform, although they may not remember doing so. Had a task been selected that was dependent on the integrity of the hippocampal formation, such as spatial navigation in the water maze, the anterograde deficit would have obscured any retention of the episode. Furthermore, temporally graded retrograde amnesia is usually seen in tasks not dependent on hippocampal integrity (Nadel \& Moscovitch, 1997).

Bilateral dorsal electrolytic lesions of the hippocampus (Kim \& Fanselow, 1992) were used to induce memory loss. This lesion technique was utilized in order to maintain congruence with previous studies demonstrating graded retrograde amnesia, most of which have used electrolytic or aspiration techniques. More specific neurotoxin- 
induced hippocampal lesions have been reported to cause temporally graded retrograde amnesia (Maren et al., 1997), but the temporal gradient is much more extended than the bulk of reports that utilize the electrolytic technique. In addition, some reports indicate that neurotoxic lesions may not induce temporally graded amnesia (Bolhuis, Stewart, \& Forrest, 1994; Mumby, Astur, Weisend, \& Sutherland, 1999). Given these considerations, electrolytic lesions were used to disrupt the dorsal hippocampus.

\section{Method}

Subjects. Fifty female Long-Evans hooded rats, between 90 and 120 days of age and weighing between 250 and $340 \mathrm{~g}$, were used in the experiment. The subjects were housed singly in hanging wiremesh cages and given ad-lib access to food and water throughout the experiment. All the procedures were conducted during the light portion of the 14:10-h day:night cycle (lights on, 0700). Three rats died during surgical procedures, and their acquisition data were eliminated from the analysis.

Apparatus. Training took place in a Y-maze, constructed according to the specifications of Gisquet-Verrier and Schenk (1994). The maze was clear Plexiglas with a grid floor, composed of $0.24-$ $\mathrm{cm}$-diameter rods spaced $9 \mathrm{~mm}$ apart. The rods were attached to a scrambler (Lafayette Instruments), which was, in turn, attached to a shock generator (Lafayette Instruments) through which a $0.2-\mathrm{mA}$ shock could be delivered. At the end of each of the 91.44-cm-long arms, but outside of the maze, was a remotely operated 40 -W light bulb. The sides of the maze were opaque, and the lids were clear to allow the procedure to be videotaped. The maze was located in a room $2.8 \times 3.3 \mathrm{~m}$. The room was dark during experimentation, except for the active light bulb.

Surgery. The rats were anesthetized with sodium pentobarbital (Nembutal; $40 \mathrm{mg} / \mathrm{kg}$ ) and placed in a stereotaxic apparatus (Kopf Instruments). Bilateral electrolytic ( $2 \mathrm{~mA} ; 10 \mathrm{sec})$ dorsal hippocampal lesions were administered according to the procedures of Kim and Fanselow (1992); the stereotaxic coordinates were the following: $2.8 \mathrm{~mm}$ posterior to bregma, $2.0 \mathrm{~mm}$ lateral to midline, and $4.0 \mathrm{~mm}$ ventral from the skull; $4.2 \mathrm{~mm}$ posterior to bregma, 3.0 lateral from midline, and 4.0 ventral from the skull's surface. A current was passed through a stainless steel lead with insulation removed from $0.5 \mathrm{~mm}$ of the end. A $2.5-\mathrm{cm}$-long incision on the dorsal medial surface of the head was held open with hemostats, and four holes were drilled in the skull. The electrode was lowered into the brain and grounded by a clip on the left hemostat. Sham-treated rats received Nembutal and no surgical manipulations. The animals recovered for 7 days before testing. Following testing, the subjects were sacrificed with an overdose of sodium pentobarbital (l cc). Their brains were perfused with saline, followed by formalin, and were frozen and sliced for histological analysis.

Procedure. The subjects were adapted to the maze 1 day before training by allowing individual rats to explore the fully lighted maze for 5 min each. On the 2 nd day, the animals were trained on the signaled avoidance task under the following procedures. The rats were placed at the end of the start arm of the maze, with the light bulb in that arm activated. The light was turned off $20 \mathrm{sec}$ later, and the bulb at the end of another arm, the safe arm for that trial, was activated, which signified that the entire grid floor, with the exception of the last $30 \mathrm{~cm}$ of the safe arm, was electrified. The task required learning to run to the end of the arm containing the lighted bulb. After $20 \mathrm{sec}$ in the safe arm, the procedure was repeated, with one of the other arms lighted. Therefore, the safe arm became the start arm for the next trial. (The sequence of correct turns was $\mathrm{L}-\mathrm{L}-\mathrm{R}-\mathrm{L}-\mathrm{R}-$ $\mathrm{R}-\mathrm{L}-\mathrm{R}-\mathrm{R}-\mathrm{L}-\mathrm{R}-\mathrm{L}-\mathrm{L}-\mathrm{R}-\mathrm{R}$.)

Each subject had 15 training trials. If correct discrimination was not achieved on the last 3 , single trials were added until three con- secutive correct choices were made. This criterion was used in order to ensure that all the subjects experienced at least 15 trials of training.

Following training, the rats were divided into five groups on the basis of their subsequent treatment. Group 1-Lesion $(n=7)$, which received the lesion no longer than $3 \mathrm{~h}$ following training (i.e., on Day 1), was expected to show amnesia for the task. Group Sham $(n=7)$ was injected with the anesthetic dose of pentobarbital following training. This group was expected to perform well. Both of these groups were tested after a 7-day recovery interval. The remaining groups were tested 37 days following training: Group 30Lesion $(n=8)$ received the lesion treatment 30 days following training and was expected to perform well, since month-old memories are typically not affected by hippocampal damage in rats (e.g., Kim \& Fanselow, 1992; Squire, 1992). Group Remind-30-Lesion ( $n=$ 9) also underwent surgery 30 days after training, but only after a reminder treatment. This reminder consisted of placing the rats in the start arm of the Y-maze for 2 min and turning the cue light on and off twice. During the reminder treatment, the rats were not exposed to any of the contingencies important for learning; the treatment was designed to "activate" the memory without promoting new learning. Group Remind-30-Lesion was expected to perform poorly if active processing renders the memories vulnerable to the amnestic treatment. Group Remind-Only $(n=9)$ was reminded without surgery, and Group Control $(n=7)$ remained in the home cage until testing. All the groups were tested with 15 trials in the Y-maze and in the same order as that at training. Errors, the dependent variable, were defined as (1) entering an unlighted arm or (2) failing to leave the start arm for the trial within $60 \mathrm{sec}$. These two types of errors were combined into a single score because both non-goal-directed escape strategies and freezing in the start arm are identical to behavior seen in initial acquisition trials in untrained rats. It was inferred that test responses that resemble behavior seen during early acquisition reflect amnesia. It should be noted that the possibility that hippocampal damage per se would affect perception, motivation, or activity at testing was evaluated by comparison with Group 30-Lesion. All the data were analyzed with a one-way analysis of variance (ANOVA) and Fisher $t$ tests. Differences were considered significant when $p<.05$. Following testing, the rats were sacrificed with an overdose of sodium pentobarbital and perfused. Their brains were frozen and sliced at $40 \mu \mathrm{m}$, and the slices were mounted and stained with cresyl violet for lesion verification.

\section{Results and Discussion}

Histology indicated that lesions were as intended, with moderate amounts of dorsal hippocampal damage (comparable with Kim \& Fanselow, 1992). The ventral hippocampus was spared, and damage to the cortex overlying the dorsal hippocampus was minimal (see Figure 1).

There were no differences among the groups in terms of errors during training [one-way ANOVA, $F(5,41)=1.712$, n.s.]; the mean training score was 8.79 errors per subject. As can be seen in Figure 2, there were significant differences in performance among the groups at testing [oneway ANOVA, $F(5,41)=5.169, p<.001]$. Sham-treated rats showed substantial savings, making an average of two errors. In contrast, Group 1-Lesion rats, which were treated immediately following training, showed amnesia for the task. They made a mean of 7.43 errors, which differed significantly from the mean of the sham-treated subjects $(p<.028)$. This finding replicates the well-established amnesia for recently acquired memories after hippocampal damage (Kim et al., 1995; Kim \& Fanselow, 1992; McClelland et al., 1995; Moscovitch, 1995; Scoville \& Mil- 


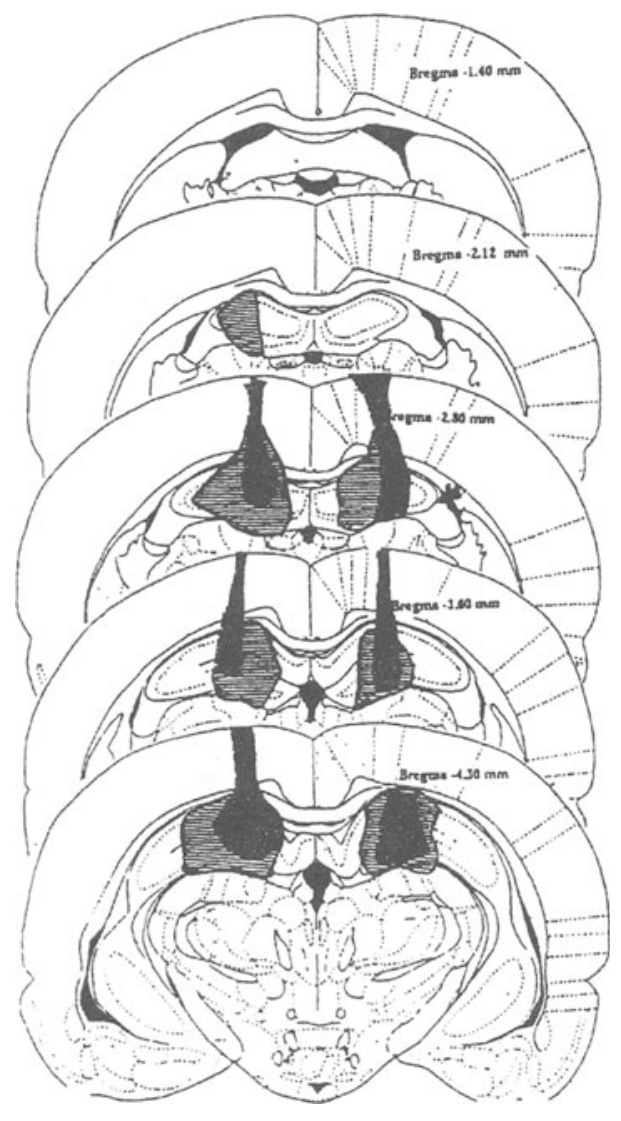

Figure 1. Largest (light shading) and smallest (dark shading) bilateral dorsal hippocampal lesions. Damage was restricted to the dorsal hippocampus, with minimal incidental cortical damage. From The Rat Brain in Stereotaxic Coordinates (Figures 24, 26, 29, 32, 35), by G. Paxinos and C. Watson, 1986, New York: Academic Press. Copyright 1986 by Academic Press. Adapted with permission.

ner, 1957; Squire, 1992; Squire \& Zola-Morgan, 1990; Winocur, 1990; Zola-Morgan \& Squire, 1992). Rats sustaining lesions 30 days following training (Group 30Lesion) performed well, with a mean of 2.6 errors, a score that did not differ from that of the Control rats $(2.28 \mathrm{er}-$ rors), which were also tested 37 days after training. This replicates the common finding that indicates the sparing of memories learned long before the lesion treatment (see Squire \& Alvarez, 1995). However, those animals reminded of the training just before the delayed lesion (Remind-30-Lesion) showed amnesia, despite the fact that the memory was the same age as that of Group 30Lesion. Remind-30-Lesion showed amnesia equivalent $(t=0.26$, n.s. $)$ to Group 1-Lesion, for which the memory was new. Remind-30-Lesion rats, with a mean error of 7, performed worse than 30 -Lesion rats $(p<.038)$, Control rats $(p<.017)$, and Remind-Only rats $(p<.004)$.

These data seem to indicate that the activity state of memory, rather than its age per se, determines vulnerability to hippocampal lesion-induced retrograde amnesia. Groups 30-Lesion and Remind-30-Lesion sustained the hippocampal treatment at the same time following learning, but only in the latter group was the memory active, and only in the latter group was amnesia evident. This outcome parallels the findings obtained with transient amnestic agents, such as ECS and hypothermia. The notion that the hippocampus is involved when memories are being actively processed is consonant with imaging studies indicating hippocampal activation upon successful memory retrieval (Nyberg, McIntosh, Houle, Nilsson, \& Tulving, 1996), a finding not immediately compatible with consolidation theory. That the hippocampus may be important for retrieving remote memories is also considered in Nadel and Moscovitch's $(1997,1998)$ recent evaluations of the status of standard models of hippocampal function in memory. Along with their proposals, the evidence from Experiment 1 indicating that presumably "consolidated" memories can be rendered amnestic by hippocampal damage presents a complication for currently accepted notions of memory processing. We emphasize that amnesia for this task probably represents an inability to retrieve the training episode, rather than the loss of the ability to perform the task at all, such as occurs with place learning (Mumby et al., 1999).

As was stated earlier, retrograde and anterograde amnesia usually show congruence, such as with loss of memory for context (e.g., Kim \& Fanselow, 1992). Although the present experiments may appear to be inconsistent with that outcome, it should be recognized that we did not evaluate anterograde memory effects of the lesion. To do so would have required a paradigm in which a separate group received a retention test subsequent to their proficient within-session learning-that is, an assessment of forgetting for information acquired after the lesion (see Spear \& Riccio, 1994, for an elaboration of this rationale). Our choice of signaled avoidance was predicated on evidence that this task can be readily acquired by hippocampal subjects (Gisquet-Verrier \& Schenk, 1994), thus permitting detection of retrograde amnesia, as measured by impairments in relearning (i.e., savings).

Again taking direction from studies exploring temporary insult-induced amnesia, Experiment 2 was conducted to explore the general implications of our observations for hippocampal function in memory. In this case, rather than investigating the susceptibility of memories to amnestic treatments, the permanence of amnesia itself was at issue.

\section{EXPERIMENT 2}

A fundamental theme in attempts to resolve the nature of retrograde amnesia has focused on the attribution of the loss to storage or retrieval deficits. Evidence supporting a retrieval view would be provided if the amnesia could be reversed or alleviated independent of new learning. Just such evidence has been obtained when agents such as ECS or hypothermia have been used to induce memory loss (Koppenaal, Jagoda, \& Cruce, 1967; Mactutus, Ferek, \& Riccio, 1980; Miller \& Springer, 1972; see Riccio \& Richardson, 1984, for a review). Recovery 


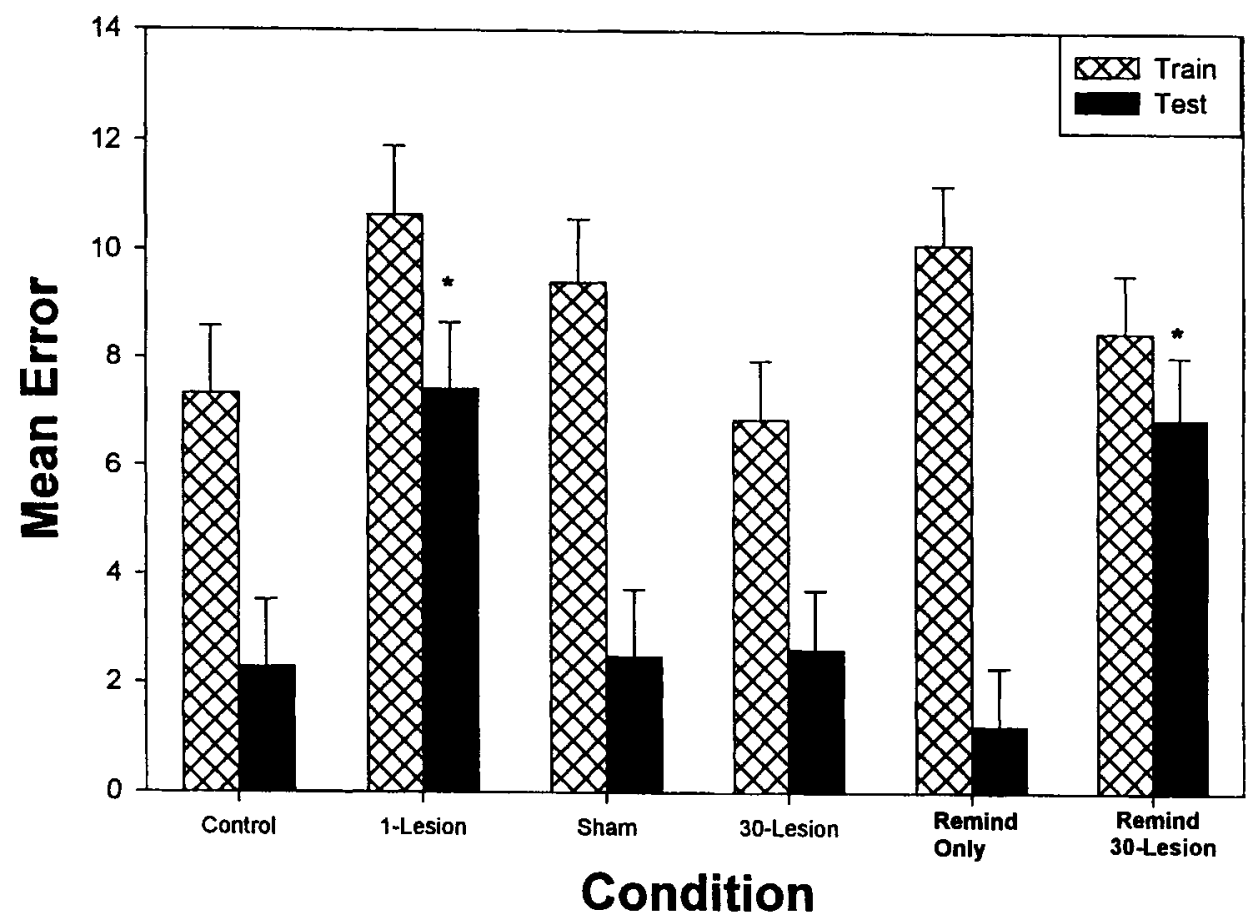

Figure 2. Experiment 1 : mean training error (hatched bars) and testing error (solid bars) represented by condition. There were no differences in the number of training errors among groups. At test, however, Groups 1-Lesion and Remind-30-Lesion showed amnesia; both of their memories were active prior to surgery. Group 30-Lesion, the memory of which was presumably inactive before the lesion, showed good memory for the task, as did Groups Control and Remind. Asterisks indicate significant difference $(p<.05)$ from comparison groups; see the text.

from amnesia was accomplished by exposing subjects to a component of the training episode prior to training (see Riccio \& Richardson, 1984, and Spear \& Riccio, 1994, for reviews). In an early influential study, Miller and Springer trained rats, using a passive avoidance paradigm, and effectively reactivated the amnestic memories with noncontingent footshock, a mild footshock delivered in a separate apparatus than that used in training. In other words, the subjects were exposed to the reinforcer in the absence of any other training stimuli. Notably, this portion of the training episode, called a reactivation treatment, is insufficient to produce new learning in naive subjects. A similar reversal of retrograde amnesia has been found with numerous behavioral tasks, including appetitive ones (Miller, Ott, Berk, \& Springer, 1974). Furthermore, Winocur and Black (1978) demonstrated a reactivation effect following hippocampal lesions, although their study concerned itself only with anterograde amnesia.

In the present experiment, the Y-maze training task was again used, following the procedure of Experiment 1, and the same lesion technique was employed. However, all the lesions occurred immediately after training, and a reactivation treatment was used just prior to testing. According to Miller and Springer (1972), an amnestic memory may be intact but difficult to access. In the present experiment, in order to promote retrieval, the reactivation treatment consisted of exposure to a noncontingent footshock in a separate room and apparatus to those used in training. Exposing subjects exclusively to the reinforcer in the absence of other cues was proposed to facilitate retrieval in those subjects retaining memory of training, without promoting learning in naive subjects.

\section{Method}

Subjects. Thirty-six female hooded Long-Evans rats were maintained under the same conditions as those detailed previously. The subjects weighed between 200 and $350 \mathrm{~g}$ and were $100-150$ days of age.

Apparatus. The same Y-maze as that described in Experiment 1 was used.

Surgery. Surgical procedures were identical to those of Experiment 1 . Histological data were equivalent to those of the first experiment and are included in the summary diagram (see Figure 1).

Procedure. The subjects were randomly assigned to five groups. Group Lesion ( $n=8$ ) sustained the hippocampal lesion within $3 \mathrm{~h}$ following training and was expected to show amnesia. Group Control $(n=6)$ did not sustain a lesion after training and was predicted to perform well. Group Lesion-Reactivation $(n=8)$ received the lesion treatment and, 7 days later, just prior to testing, was given a noncontingent footshock in a separate apparatus as a reactivation treatment, following the strategy of Miller and Springer (1972). If hippocampal lesion-induced amnesia is reversible, this group would be expected to perform well. To ascertain the effects of the reactivation treatment itself, Group Reactivation $(n=7)$ experienced the noncontingent footshock before testing, a treatment that was pre- 
dicted to have no effect (since forgetting typically does not occur following a 7-day training-to-test interval, resulting in ceiling performance) or to cause a mild improvement in performance. In addition, in order to ensure that the noncontingent footshock did not produce a training effect, an untrained group (Untrained-Reactivation; $n=7$ ) was given the reactivation treatment prior to their first exposure to the maze. This group was expected to perform poorly.

The rats were trained in the Y-maze in the same manner as that in Experiment $l$ and then were either removed from the maze and returned to the home cage or taken to the surgery room, where the lesion procedure took place. Seven days later, the subjects in the three reactivation conditions (Lesion-Reactivation, Reactivation, and Untrained-Reactivation) were transported to the reactivation room, held for $30 \mathrm{sec}$ on the arm of the experimenter, and placed into the reminder box. There, they experienced one $3-\sec 0.1-\mathrm{mA}$ footshock, were taken to the Y-maze, and were tested for retention of the task as previously described. The rats in the nonreactivation conditions (Control and Lesion) were placed into the reactivation chamber with no shock presented before being tested in the Y-maze in order to equate transportation and handling.

A one-way ANOVA and planned comparisons using Fisher tests were used to confirm differences among the groups.

\section{Results and Discussion}

There were no differences in errors among the groups during training $[F(3,24)=0.89$, n.s.; four groups that experienced training, 29 subjects], or in number of trials to criterion $[F(3,24)=0.57$, n.s. $]$. Figure 3 shows the differences evident among the groups during testing $[F(3,31)=$ $24.65, p<.001]$. Group Control and Group Reactivation performed well, making an average of 2.5 and 2.1 errors, respectively. As was expected, and corroborating the results of Experiment 1, Group Lesion demonstrated poor retention, with an average of 8.4 errors, which differed reliably from the scores of Groups Control and Reactivation $(p s<.001)$. However, lesion rats receiving a pretest reactivation treatment (Group Lesion-Reactivation) performed well, with an average of 3.6 errors, a score significantly different from Group Lesion $(p<.002)$, but not different from Groups Control and Reactivation. It thus appears that the noncontingent footshock was effective at reminding lesion animals of the task, reversing the amnesia induced by hippocampal damage evident in the nonreminded group (Group Lesion). Group UntrainedReactivation, which experienced only the reactivation treatment prior to "testing," performed significantly more poorly than any other group (vs. Group LesionReactivation, $p<.001$; vs. Group Lesion, $p<.03$ ), suggesting that, in the absence of training, a reactivation treatment may actually retard performance.

In order to determine whether amnesia for the training was complete, the number of errors that each group made in the first 15 training trials was compared with the number of errors made during testing. Figure 4 indicates that all groups showed a significant decrease in errors from training to testing, except for Group Lesion, the error scores of which were not different between training and test [Control: $F(1,10)=7.74, p<.019$; Lesion-Reactivation: $F(1,14)=52.32, p<.0001$; Reactivation: $F(1,10)=$ $14.51, p<.003$; Lesion: $F(1,14)=2$, n.s.]. In operational terms, therefore, the Lesion Group animals behaved as though they were naive to the task, making an equivalent number of errors at training and testing.

These results indicate that, like transient insult-induced amnesia, hippocampal lesion-induced retrograde amnesia may be attenuated or eliminated by a reactivation treatment that does not produce new learning. When amnestic subjects were provided with appropriate information-in this case, the reinforcer used in training - retrieval of the target memory was successful. These data are consonant with theories noting that retrieval is superior when attributes of the training and testing contexts are similar (Spear, 1973; Spear \& Riccio, 1994; Tulving, 1974; Tulving \& Thompson, 1973). The performance of reminded subjects contrasts sharply with the performance of those only sustaining the lesion, despite the fact that the two groups underwent identical lesion treatments.

Presumably, the reactivation treatment allowed rats to retrieve the memory of the Y-maze training and utilize it as they were tested. Why, then, did the first training trial not serve to reactivate the memories of other amnestic rats in either experiment? This is an issue that has emerged in other studies using relearning (savings) measures to assess reactivation treatments (Gordon, McCracken, DessBeech, \& Mowrer, 1981), and a clear explanation has not been identified. The most reasonable account may be that memory reactivation is possible if enough time is available to allow assimilation of information. For example, in the present study, the reactivation treatment occurred in a different room, allowing the rats about $3 \mathrm{~min}$ to "process" the treatment prior to placement in the Y-maze. Nonreactivated rats, on the other hand, were placed immediately into the maze and exposed to a quick succession of task demands. It is possible that this demand for rapid and appropriate action did not allow the subjects time to retrieve the memory and/or appropriately use any retrieved information. Again, the reactivation treatment clearly did not result in new learning, as can be seen in the performance of Group Untrained-Reactivation.

It appears that in lesion subjects, the information is retained, but the retrieval process is disrupted, a finding mirroring earlier ones regarding amnesia induced by transient amnestic treatments (Koppenaal et al., 1967; Mactutus et al., 1980; Miller \& Springer, 1972; Riccio \& Richardson, 1984). Such an impairment is consistent with recent work by Holt and Maren (1999) implicating the hippocampus in retrieval of memory for a fear-related context. Although amnesia is sometimes more severe with less complete hippocampal damage (Bachevalier \& Mishkin, 1989), it is possible that the sparing of the dorsal hippocampus led to the ability to reactivate memory. Another possible explanation for the efficacy of our reactivation treatment is that it gained access to the representation of the training episode via a different route, perhaps involving the amygdala (Baker, Kesner, \& Michal, 1981). The system subserving the emotional/fearful aspect of footshock, which presumably involves amygdala, was not damaged by the hippocampal lesion. When rats with lesions are placed in the maze at test, the context does not 


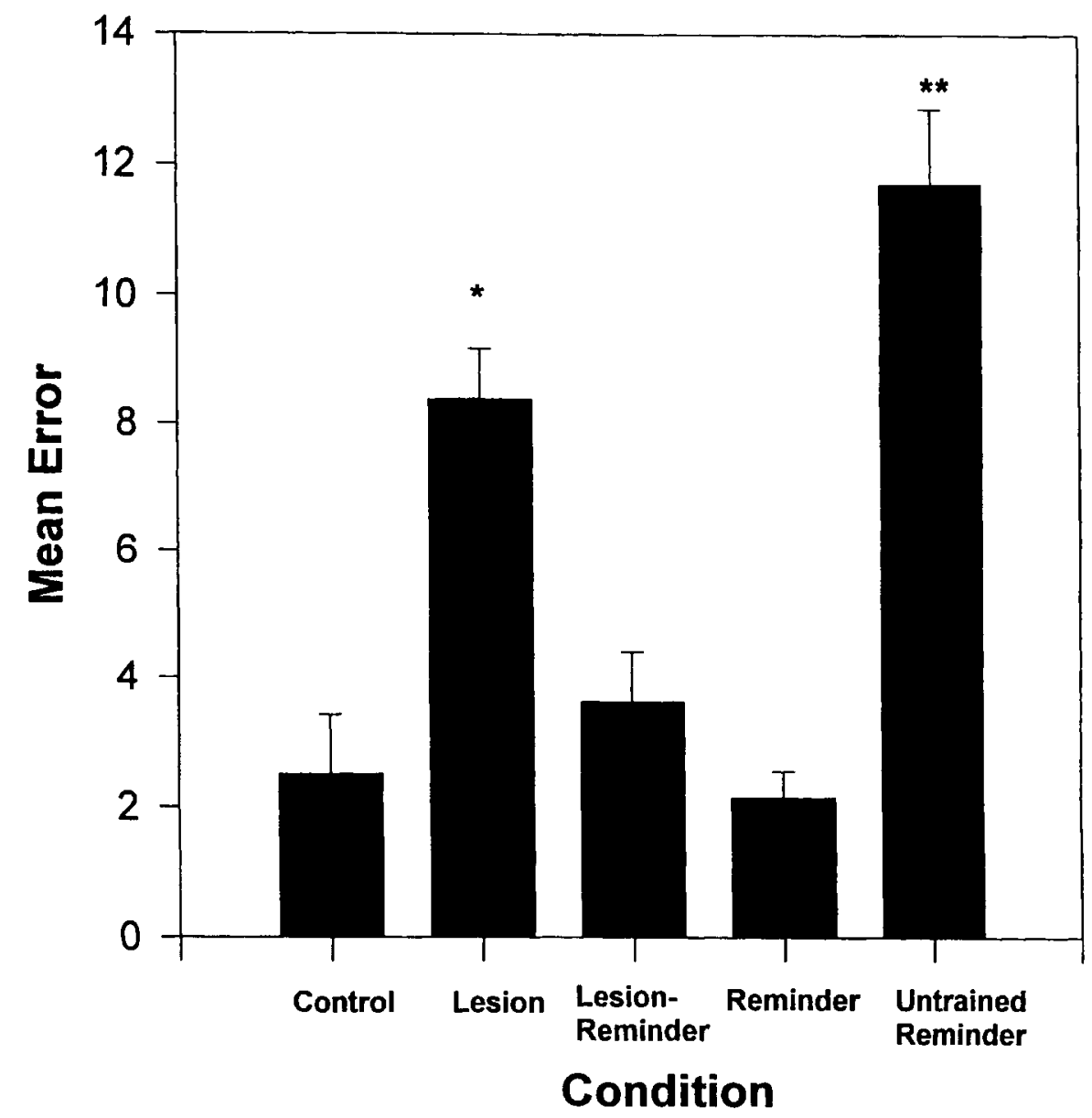

Figure 3. Experiment 2: Group Lesion evidenced amnesia for the task, unlike Group Lesion-Reactivation, which was exposed to a cuing treatment prior to testing that reversed the amnesia. Groups Control and Reactivation showed good retention of the task. The score of the untrained Group Reactivation-Only demonstrated that the cuing treatment did not induce new learning.

afford retrieval of the memory, and they therefore show amnesia. However, if they are first exposed to the footshock in the reactivation situation, they then have an opportunity to access the representation through information not dependent on the hippocampus but, instead, dependent on an intact amygdala. This interpretation, although speculative, is congruent with evidence of intact fear conditioning in hippocampal animals, but not in those with amygdala damage (Baker et al., 1981).

\section{GENERAL DISCUSSION}

It could be argued that the lesions in the present studies, which preserved some hippocampal tissue, did not fully eradicate the engram or completely disrupt the consolidation process, allowing sparing of memory. However, we believe that such an interpretation of the data presented here is implausible for several reasons. First, the animals in the nonreminded lesion groups in both studies demonstrated complete retrograde amnesia, as measured by task performance. Along with appropriate histological verification, the existence of clear amnesia has historically been evidence of effective lesion treatment (see Kim \& Fanselow, 1992; Kim et al., 1995; McClelland et al., 1995; Squire, 1992; Winocur, 1990; Zola-Morgan \& Squire, 1992). Since both the lesions and the amnesia in the present study mimic those of previous investigations, it seems important to ask whether reactivation treatments could have reversed amnesia in previously reported cases.

Furthermore, a number of investigations have demonstrated that partial damage is just as, if not more, disruptive for memory than is complete damage (e.g., Bachevalier \& Mishkin, 1989; Gaffan, 1997). Perhaps more important, a criticism based on insufficient lesions could possibly apply only to the second experiment reported here, in which an amnestic memory was recovered through a reactivation treatment. In order to discount the results of Experiment 1, where an older and presumably established memory was rendered vulnerable to amnesia by giving subjects a reminder exposure, it is necessary to pro- 


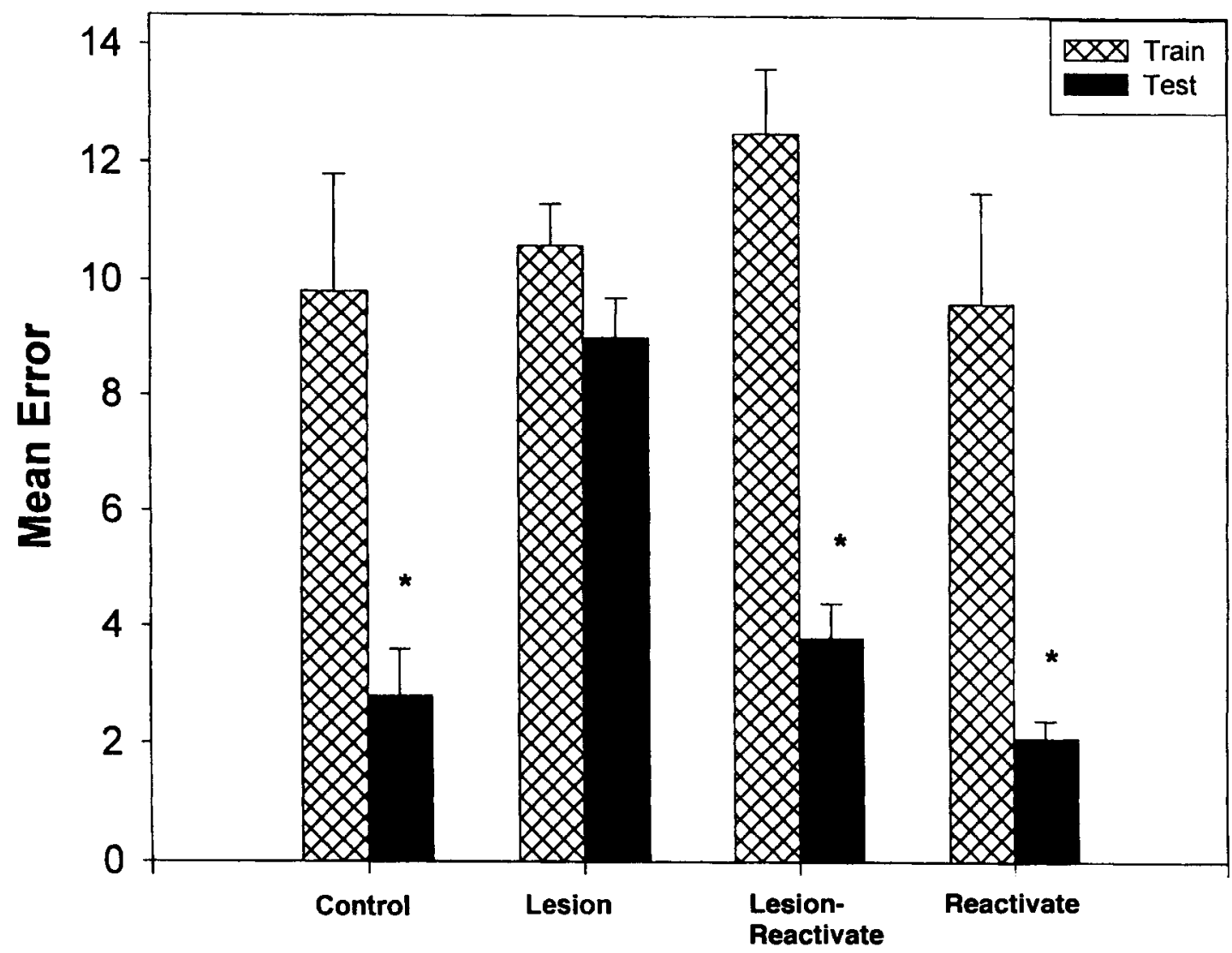

\section{Condition}

Figure 4. Experiment 2: A comparison of the first 15 training trials (solid bars) with the 15 test trials (hatched bars) demonstrated the complete amnesia of Group Lesion, the scores of which were indistinguishable between training and testing. Groups Control, Lesion-Reactivation, and Reactivation showed considerable task retention.

pose the opposite- that is, that amnesia results from too much damage.

Despite increasingly specific elaborations of the functional neuroanatomy of the medial temporal lobe components and the parahippocampal cortex, the hippocampus proper is still considered crucial for normal anterograde and retrograde episodic memory function (Aggleton \& Brown, 1999; Cohen \& Eichenbaum, 1993). It is therefore suggested here that although electrolytic lesions do not produce specialized damage, they can nonetheless allow inferences with respect to hippocampal functioning in memory.

The finding in Experiment 1, that reexposure to training cues can make old memory vulnerable to hippocampal damage, not only extends earlier studies using transient amnestic treatments (e.g., ECS, hypothermia), but also adds to a growing body of research on the consequences of reactivation. For example, Sara and her colleagues have found that well-established memories made active by cuing can be disrupted in a time-dependent manner by propanolol, a beta-adrenergic blocker (Przybyslawski, Roullet, \& Sara, 1999; Przybyslawski \& Sara, 1997). Conversely, as Rodriguez and his colleagues have shown, agents, such as glucose and cocaine, that can enhance new memories also do so when administered at the time of a cuing exposure designed to reactivate an earlier memory (Rodriguez, Horne, \& Padilla, 2000; Rodriguez, Rodriguez, Phillips, \& Martinez, 1993; Rodriguez, Van Ausdle, Dhanens, \& Mondragon, 1993).

Because the lesions here were produced electrolytically, it might be suggested that our findings represent a form of ECS-induced amnesia, rather than a lesion effect per se. That interpretation is unlikely for two reasons. First, the argument may be applied to the finding that memory reactivation results in amnesia (Experiment 1) but can hardly account for Experiment 2, where amnesia was alleviated despite destruction of dorsal hippocampal tissue. Furthermore, it is well established that ECS and electrical brain stimulation can produce amnesia only within an extraordinarily brief period following acquisi- 
tion. When there are several hours between training and the lesion treatment, as in our experiments, amnesia produced by ECS is not obtained.

These results do appear to be in contrast to the apparently irreversible nature of human amnesia. Nonetheless, there is at least some evidence that humans with lesions also show reversal of amnesia when exposed to a particularly salient reminder cue (Lucchelli, Muggia, \& Spinnler, 1995). In addition, neuropsychological data indicate that retrograde amnesia in humans may "shrink" (Benson \& Geshwind, 1967); that is, the period of time affected by the amnesia progressively shortens, allowing patients to recall information for which they were initially amnestic. Perhaps it should not be surprising that the reminder effect is not more well established in humans, given the uncontrolled nature of human amnestic testing. Whereas animal subjects can be exposed to a small number of life events and later be given reminder cues tailored to those events, this cannot be readily achieved with human retrograde amnesia.

Taken together, the results of the present experiments are difficult to reconcile with established characteristics of retrograde amnesia produced by hippocampal damage. Experiment 1 demonstrated that presumably "consolidated" memories are vulnerable to hippocampal damage, an outcome difficult even for current reconceptualizations of hippocampal function in memory (e.g., Nadel \& Moscovitch, 1997) to accommodate. The results of Experiment 2 showed that an amnesia normally attributed to consolidation failure is reversible. Especially with regard to Experiment 2, theories capable of accommodating these anomalous results by emphasizing the importance of the hippocampus in retrieval (Holt \& Maren, 1999; Nadel \& Moscovitch, 1997) are likely to promote a better understanding of the intricate involvement of the hippocampus in memory processing.

\section{REFERENCES}

Aggleton, J. P., \& Brown, M. W. (1999). Episodic memory, amnesia, and the hippocampal-anterior thalamic axis. Behavioral \& Brain Sciences, 22, 425-489.

anagnostaras, S. G., Maren, S., \& Fanselow, M. S. (1999). Temporally graded retrograde amnesia of contextual fear after hippocampal damage in rats: Within-subjects examination. Journal of Neuroscience, 19, 1106-1114.

BaChevalier, J., \& Mishkin, M. (1989). Mnemonic and neuropathological effects of occluding the posterior cerebral artery in Macaca mulatta. Neuropsychologia, 27, 83-105.

BaKer, L. J., Kesner, R. P., \& Michal, R. E. (1981). Differential effects of a reminder cue on amnesia induced by stimulation of amygdala and hippocampus. Journal of Comparative \& Physiological Psychology, 95, 312-321.

BENSON, D. F., \& GeSHWIND, N. (1967). Shrinking retrograde amnesia. Journal of Neurology, Neurosurgery, \& Psychiatry, 30, 539-544.

Bolhuis, J. J., Stewart, C. A., \& Forrest, E. M. (1994). Retrograde amnesia and memory reactivation in rats with ibotenate lesions to the hippocampus or subiculum. Quarterly Journal of Experimental Psychology, 47, 129-150.

Bures, J., \& Buresova, O. (1963). Cortical spreading depression as a memory disturbing factor. Journal of Comparative \& Physiological Psychology, 56, 268-272.
CoHEN, N. S. (1984). Preserved learning capacity in amnesia: Evidence for multiple memory systems. In L. R. Squire \& N. Butters (Eds.), Neuropsychology of memory (pp. 83-103). New York: Guilford.

Cohen, N. S., \& Eichendaum, H. (1993). Memory, amnesia and the hippocampal system. Cambridge, MA: MIT Press.

DUNCAN, C. P. (1949). The retroactive effect of electroshock on learning. Journal of Comparative \& Physiological Psychology, 42, 32-44.

GafFan, D. (1997). Episodic and semantic memory and the role of the not-hippocampus. Trends in Cognitive Sciences, 1, 246-248.

Gisquet-VerRier, P., \& Schenk, F. (1994). Selective hippocampal lesions in rats do not affect retrieval processes promoted by prior cuing with the conditioned stimulus or the context. Psychobiology, 22, 289-303.

Gordon, W. C., McCracken, K. M., Dess-Beech, N., \& Mowrer, R. R. (1981). Mechanisms for the cuing phenomenon: The addition of the cuing context to the training memory. Learning \& Motivation, 12, 196-211.

HeBB, D. O. (1949). The organization of behavior. New York: Wiley

Holt, W., \& MAREN, S. (1999). Muscimol inactivation of the dorsal hippocampus impairs contextual retrieval of fear memory. Journal of Neuroscience, 19, 9054-9062.

KeSNER, R. P., \& Doty, R. W. (1968). Amnesia produced in cats by local seizure activity initiated from the amygdala. Experimental Neurology, 21, 58-68.

Kim, J. J., Clark, R. E., \& Thompson, R. F. (1995). Hippocampectomy impairs the memory of recently, but not remotely acquired trace eyeblink conditioned responses. Behavioral Neuroscience, 9, 195-203.

KIM, J. J., \& FANSELOW, M. S. (1992). Modality-specific retrograde amnesia of fear. Science, 256, 675-677.

KoppenAal, R. J., JAGODA, E., \& CRUCE, J. A. F. (1967). Recovery from ECS-induced amnesia following a reminder. Psychonomic Science, 9, 293-294.

Lucchell, F., Muggia, S., \& Spinnler, H. (1995). The "Petites Madeleines" phenomenon in two amnesic patients: Sudden recovery of forgotten memories. Brain, 118, 167-183.

Mactutus, C. R., Ferek, J. M., \& Riccio, D. C. (1980). Amnesia induced by hyperthermia: An unusually profound, yet reversible, memory loss. Behavioral \& Neural Biology, 30, 260-277.

Mactutus, C. R., \& Riccio, D. C. (1978). Retrograde amnesia for old (reactivated) memory: Some anomalous characteristics. Science, 204, 1319-1320.

Maren, S., Aharonov, G., \& Fanselow, M. S. (1997). Neurotoxic lesions of the dorsal hippocampus and Pavlovian fear conditioning in rats. Behavioural Brain Research, 88, 261-274.

McClelland, J. L., MCNaughton, B. L., \& O'Reilly, R. C. (1995). Why there are complementary learning systems in the hippocampus and neocortex: Insights from the successes and failures of connectionist models of learning and memory. Psychological Review, 102 419-457.

McGaUGH, J. L. (1966). Time-dependent processes in memory storage. Science, 153, 1351-1358.

Miller, R. R., Ott, C. A., Berk, A. M., \& Springer, A. D. (1974). Appetitive memory restoration after electroconvulsive shock in the rat. Journal of Comparative \& Physiological Psychology, 87, 717723.

Miller, R. R., \& SPRINGER, A. D. (1972). Induced recovery of memory in rats following electroconvulsive shock. Physiology \& Behavior, 8, 645-651.

Misanin, J. R., Miller, R. R., \& Lewis, D. J. (1968). Retrograde amnesia produced by electroconvulsive shock after reactivation of a consolidated memory trace. Science, 160, 554-555.

Moscovitch, M. (1995). Recovered consciousness: A hypothesis concerning modularity and episodic memory. Journal of Clinical \& Experimental Neuropsychology, 17, 276-290.

Mumby, D. G., Astur, R. S., Weisend, M. P., \& Sutherland, R. J. (1999). Retrograde amnesia and selective damage to the hippocampal formation: Memory for places and object discriminations. Behavioural Brain Research, 106, 97-107.

NADEL, L., \& Moscovitch, M. (1997). Memory consolidation, retrograde amnesia and the hippocampal complex. Current Opinion in Neurobiology, 7, 217-227. 
Nadel, L., \& Moscovitch, M. (1998). Hippocampal contributions to cortical plasticity. Neuropharmacology, 37, 431-439.

Nyberg, L., McIntosh, A. R., Houle, S., Nilsson, L.-G., \& TulvING, E. (1996). Activation of medial temporal lobe structures during episodic memory retrieval. Nature, 380, 715-717.

Patterson, K. E., Lawler, E., \& Rochester, S. R. (1978). Retrograde amnesia from electroconvulsive therapy. Neuropsychologia, 16, 91 . 97.

Paxinos, G., \& Watson. C. (1986). The rat brain in stereotaxic coordinates. New York: Academic Press.

Przybyslawski, J., Roullet, P., \& Sara, S. J. (1999). Attenuation of emotional and nonemotional memories after their reactivation: Role of beta adrenergic receptors. Journal of Neuroscience, 19, 6623-6628.

Przybyslawski, J., \& SARA, S. J. (1997). Reconsolidation of memory after its reactivation. Behavioural Brain Research, 84, 24-26.

RiBor, T. (1882). The diseases of memory. New York: Humboldt.

Riccio, D. C., Hodges, L. A., \& Ranidall, P. K. (1968). Retrograde amnesia produced by hypothermia in rats. Journal of Comparative \& Physiological Psychology, 66, 618-622.

Riccio, D. C.. \& Richardson, R. (1984). The status of memory following experimentally induced amnesias: Gone, but not forgotten. Phusiological Psychology, 12, 59-72.

Rodriguez, W. A., Horne, A. C., \& Padilla, J. L. (2000). Effects of glucose and fructose on recently reactivated and recently acquired memories. Progress in Neuropsychopharmacology \& Biological Psychiatry, 23, 1285-1317.

Ronkiguez, W. A., Rodriguez, S. B., Phillips, M. Y.. \& Martinez. J. L. (1993). Post-reactivation cocaine administration facilitates later acquisition of an avoidance response in rats. Behavioural Brain Research, 59, 125-129.

Rodriguez, W. A., Van ausdle, L. R., Dhanens, K., \& Mondragon. A. N. (1993). Glucose modulates recently reactivated memories. Psychobiology, 21, 93-100.

SCOVILLE, W. B.. \& MILNER. B. (1957). Loss of recent memory after bilateral hippocampal lesions. Journal of Neurology. Neurosurgery, \& Psvchiatry, 20, 11-21.
Silveria, J. M., \& Kimble. D. P. (1968). Brightness discrimination and reversal in hippocampally-lesioned rats. Physiology \& Behavior, 3 , 625-630.

SPEar, N. E. (1973). Retrieval of memory in animals. Psychological Review, 80, 163-194.

SPEAR, N. E., \& Riccio, D. C. (1994). Memory: Phenomena and principles. Boston: Allyn \& Bacon.

SquiRE, L. R. (1992). Memory and the hippocampus: A synthesis of finding with rats, monkeys, and humans. Psychological Review, 99, 195-231.

Squire, L. R., \& Alvarez, P. (1995). Retrograde amnesia and memory consolidation: A neurobiological perspective. Current Opinion in Neurobiology, 5, 169-177.

SquiRe, L. R., \& Kandel, E. R. (1999). Memory: From mind to molecules. New York: Scientific American Library.

Souire, L. R., \& Zol.a-Morgan, S. (1990). The medial temporal lobe memory system. Science, 253, 1380-1386.

Tulving, E. (1974). Cue-dependent forgetting. American Scientist, 62 , 74-82.

Tulving, E., \& Thompson, D. M. (1973). Encoding specificity and retrieval processes in episodic memory. Psychological Review, 80, 352373.

WINOCUR, G. (1990). Anterograde and retrograde amnesia in rats with dorsal hippocampal or dorsomedial thalamic lesions. Behavioural Brain Research, 38, 145-154.

WinocuR, G., \& BLACK, A. H. (1978). Cue-induced recall of a passive avoidance response by rats with hippocampal lesions. Physiology \& Behavior, 21, 39-44.

Zola-Morgan, S.. \& SQuire, L. R. (1992). The primate hippocampal formation: Evidence for a time-limited memory storage. Science, 250, 288-290.

(Manuscript received February 7, 2000; revision accepted for publication June 7,2000 .) 